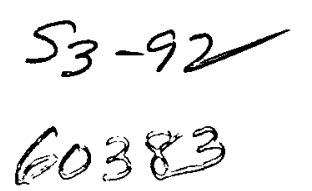

\title{
VORTICITY AND DIVERGENCE IN THE SOLAR PHOTOSPHERE
}

\author{
YI Wang, ${ }^{1}$ Robert W. Noyes, ${ }^{1,2}$ Theodore D. Tarbell, ${ }^{3}$ and Alan M. Title ${ }^{3}$ \\ Receired 1994 November 7: accepted 1995 January 17
}

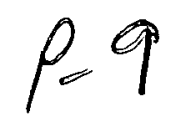

\begin{abstract}
We have studied an outstanding sequence of continuum images of the solar granulation from Pic du Midi Observatory. We have calculated the horizontal vector flow field using a correlation tracking algorithm, and from this determined three scalar fields: the vertical component of the curl, the horizontal divergence, and the horizontal flow speed. The divergence field has substantially longer coherence time and more power than does the curl field. Statistically, curl is better correlated with regions of negative divergence-that is, the vertical vorticity is higher in downflow regions, suggesting excess vorticity in intergranular lanes. The average value of the divergence is largest (i.e., outflow is largest) where the horizontal speed is large; we associate these regions with exploding granules. A numerical simulation of general convection also shows similar statistical differences between curl and divergence. Some individual small bright points in the granulation pattern show large local vorticities.
\end{abstract}

Subject headings: hydrodynamics-Sun: atmosphere - Sun: granulation

\section{INTRODUCTION}

Motions of magnetic footpoints are thought to be closely related to heating in the upper solar atmosphere (Sturrock \& Uchida 1981; Zirker \& Cleveland 1993). Motions of magnetic fields appear to be closely related to convective flows in the photosphere, as revealed by the continuously changing patterns of solar granulation. Studies of the flows in the photosphere are important for obtaining a better understanding of the magnetic heating process. Other stars on and off lower main sequence, which exhibit all the phenomena of solar magnetic activity and atmospheric heating, have atmospheres that are probably energized in a very similar way. Yet the Sun is the only star for which we have the possibility, in the foreseeable future, to study the interaction of convection and magnetic fields at the relevant spatial scale-which in the solar photosphere is roughly one density scale height, of the order $100 \mathrm{~km}$. Therefore detailed study of the flow fields in the solar photosphere can provide us with important insights about the outer atmosphere of stars.

Only with data of the highest spatial resolution free of atmospheric seeing distortions can we determine the true horizontal flow field in the solar photosphere. Though limited in the number of images and duration, such a data set was obtained by the Solar Optical Universal Polarimeter (SOUP) aboard the 1985 Spacelab 2 space shuttle flight (Title et al. 1986). These images have been used to study the statistical properties of solar granulation (Title et al. 1989), the transport of magnetic fields by horizontal flows (Simon et al. 1988), and temporal properties of horizontal velocities and their associated diver- gence and curl (Tarbell et al. 1992). Aside from the abovementioned data set, no other time series of high-resolution images from space is available. However, ground-based data

\footnotetext{
' Department of Astronomy, Harvard University, 60 Garden Street. Cambridge, MA 02138

2 Smithsonian Astrophysical Observatory, 60 Garden Street, Cambridge, MR 02138.

${ }^{3}$ Lockheed Palo Alto Research Laboratories, 3251 Hanover Street. Palo Alto. CA 943041121.
}

can in principle yield higher resolution (because of the larger optics of ground-based telescopes) provided the degradation caused by seeing can be overcome. Destretching can be used to remove atmospheric seeing distortions in ground-based images (November 1986; Topka, Tarbell, \& Title 1986). Destretched high-resolution images from Pic du Midi observatory in France, and from the Swedish Solar Telescope at La Palma in the Canary Islands have become valuable tools for studying dynamics at the solar surface.

The rich dynamics of the solar photosphere makes the extraction of flow fields a complicated process even with seeing-free data. Solar $p$-mode oscillations, surface waves, motions regulated by the magnetic field, motions due to mesogranulation and supergranulation, all enter into the picture we are trying to understand. The subfundamental Fourier filter (Title et al. 1992) developed at Lockheed Palo Alto Research Laboratories (LPARL) has been applied to both ground-based data and those obtained in space to remove the effect of solar oscillations. A filtered high-resolution movie shows high stability with little effects from solar oscillations and is suitable for studying horizontal flows in the solar surface.

An effective method for studying horizontal motions at the solar surface is local correlation tracking (LCT; November \& Simon 1988). This is an extension of "destretching" of successive solar images to remove distortions induced by atmospheric seeing. LCT uses a finer grid to track solar features and calculate their frame-to-frame motion. Unfortunately, the image motion caused by seeing is indistinguishable from solar flows in a single measurement. The seeing distortion has a correlation time of a fraction of a second, and appears as a "white" noise source with a flat temporal spectrum. Therefore, if the solar motions are oversampled in time, the seeing distortions can be reduced by simple averaging. This-process however is complicated by the evolution of solar features, most notably granules, with characteristic timescales of a few minutes. The method is most effective with data sets whose cadence is short compared to that timescale.

Using images taken at Pic du Midi Observatory and processed at LPARL, Muller \& Roudier (1992) have studied the evolution of network bright points (NBP), and Muller et al. 
(1992) have studied evolution and advection of mesogranulation. Brandt et al. (1988) have found vortex flows on a scale larger than the granulation in a sequence of images obtained at the Swedish Solar Telescope in La Palma. Similar data have also been used in modeling exploding granules (Simon, Title, \& Weiss 1991).

In this paper we study three scalar fields in the photosphere, using images obtained at Pic du Midi Observatory. We calculate the vertical component of the vorticity, $(\nabla \times v)_{z}$, which we call Curl, the divergence of the horizontal flow $\left(\nabla \cdot v_{h}\right)$, called Div, and horizontal flow speed $\left(\left|v_{k}\right|\right)$, called speed. A convection simulation of Porter, Woodward, \& Mei (1991) is also analyzed, using the same analysis techniques. The Pic du Midi data set is among the best available, although it suffers from lack of correlative line of sight velocity and magnetic data, with limited linearity, being digitized from photographic plates. Destretching is first used to reduce distortions due to " seeing" between successive frames. Then a subfundamental filter is applied to one of the data sets to remove motions due to solar $p$-mode oscillations and further reduce the noise level. These processed images can then be used to derive horizontal flows associated with solar convection using LCT.

The next section provides detailed information on the data used in this paper and discusses the sources of noise in these data sets. Section 3 deals with the horizontal vector flow fields and associated flow speed distribution. Section 4 discusses the spatial and temporal properties of Div and Curl fields derived from the flow fields, while the relationship between Div and Curl fields is discussed in $\S 4$. In $\S 5$, we discuss high-velocity rotational motion of a pair of NBPs in the Pic du Midi data. Further discussion and conclusions are given in $\$ 6$.

\section{DATA AND ANALYSIS}

Three sets of data are studied: two subsets of the Pic du Midi data called WL and GD, and a nonlinear compressible threedimensional simulation of generalized convection. The rationale for analyzing three separate data sets is as follows: the WL data were chosen because they have been subfundamental filtered to remove the effects of the 5 minute oscillation, thus revealing the residual convective flow field more clearly. Because of concern that the filtering process might affect the apparent properties of the residual flow field, we also analyzed the GD data set, which is an unfiltered (and longer) data set from the same observing sequence. The simulation data set was analyzed to see to what extent our findings are also present in numerical simulations of convection, and thus whether our findings are general properties of convection or specific to solar conditions. The WL data set, however, serves as the primary data set in this study. Table 1 provides details of the data sets.

1. The WL data set contains 526 continuum ( $5750 \AA$ ) ultrahigh-resolution images near disk center, taken by $R$. Muller at Pic du Midi Observatory with the $50 \mathrm{~cm}$ refractor, on 1988 September 20 . Every 20 seconds a set of 50 burst images was taken by a high-speed film camera (Muller \& Roudier 1992). One frame with the best image quality over a selected region is selected in each burst set. Thus on average each successive frame in the WL series is separated by 20 seconds. Seeing was excellent throughout the observation and diffraction limit was approached across the field of view in almost every selected frame. The WL series was digitized at LPARL at 0.065 arcsec pixel ${ }^{-1}$, with $1024^{2}$ pixel $\left(66.56^{2}\right.$ arcsec $\left.^{2}\right)$ in the field, spanning 2 hr 55 minutes. We have analyzed a subsection of the $\mathrm{WL}$ series containing $480^{2}$ pixel $\left(31.2^{2}\right.$ arcsec $\left.{ }^{2}\right)$ in size, spanning 57 minutes. These images were rigidly aligned, and then destretched to remove atmospheric seeing distortions. The subfundamental filter was then applied to remove brightness variations due to the 5 minute p-mode oscillations.

This portion of the WL data set has been analyzed before by Muller \& Roudier (1992). Muller et al. (1992) also analyzed the full WL data set and generated flow maps using LCT. We independently used LCT to calculate the horizontal flow fields from the positional difference of the local brightness pattern between two image frames, but at different resolution from Muller et al. The window for our flow maps is a truncated Gaussian with FWHM of 10 pixels $(0.65$ arcsec) and a cutoff of 20 pixels. The spacing between the location of the flow field vectors is 10 pixels.

2. The comparison GD data set had previously been digitized from the same raw data set as was used for the WL data, but for another purpose. Compared to the WL data, the spatial position of the GD data was slightly different, the pixel size about double ( 0.1415 arcsec), and the number of pixels in each direction (512) half as great, so that the area covered $\left(72.45^{2}\right.$ $\operatorname{arcsec}^{2}$ ) was similar. The GD data had been aligned and destretched. However, the data are substantially more noisy than the WL data due to lack of Fourier filtering. Flow maps had also been generated for the GD data set using LCT. The resolution of these flow maps corresponds to a truncated Gaussian with FWHM of 1.132 arcsec (8 pixels). While the portion of WL data set we used has significantly better spatial resolution and signal-to-noise ratio (SNR), the GD data set is 3 times longer in time.

3. We have studied a three-dimensional compressible convection simulation set calculated using the Piecewise-Parabolic Method (Porter, Woodward, \& Mei 1991), provided to us by N. Hurlburt. The simulation spanned six pressure scale beights and had an array size in $x, y$, and time of $(200,200,100)$. The numerical scheme solves the inviscid Euler equations, and hence has no formally defined viscosity. Thus the Rayleigh and Reynolds numbers are formally infinite. While the simulation is not meant to reproduce solar conditions, nevertheless it does allow us to compare our findings with some general properties of convective flows. The flow fields provided in the simulation are noise-free.

Each flow field in a given set of data will have three associated scalar fields: Div, Curl, and speed. The Div and Curl,

TABLE 1

INFORMATON FOR EACH DATA SET UsED

\begin{tabular}{ccccccc}
\hline \hline Data Sets & $\begin{array}{c}\text { Pixel } \\
\text { Resolution }\end{array}$ & $\begin{array}{c}\text { Time } \\
\text { Resolution (s) }\end{array}$ & $\begin{array}{c}\text { Flow } \\
\text { Resolution }\end{array}$ & $\begin{array}{c}\text { Size of Arrays } \\
(x, y, t)\end{array}$ & $\begin{array}{c}\text { Field of } \\
\text { View }\end{array}$ & Duration \\
\hline Pic du Midi. WL ...... & 0.065 & 20 & $0: 65$ & $(480,480,172)$ & $31: 20$ & 57 minutes \\
Pic du Midi, GD..... & $0: 1415$ & 20 & 17132 & $(512,512,525)$ & $72: 45$ & $2 \mathrm{hr}, 55$ minutes \\
\hline
\end{tabular}


fields are calculated in the following way:

$$
\begin{aligned}
\text { Div }=\frac{\hat{\partial} v_{x}}{\partial x}+\frac{\hat{c} v_{y}}{\hat{c} y}= & \frac{v_{x}(x+\Delta x)-v_{x}(x-\Delta x)}{2 \Delta x} \\
& +\frac{v_{y}(y+\Delta y)-v_{y}(y-\Delta y)}{2 \Delta y} \\
\text { Curl }=\frac{\partial v_{y}}{\partial x}-\frac{\partial v_{x}}{\partial y}= & \frac{v_{y}(x+\Delta x)-v_{y}(x-\Delta x)}{2 \Delta x} \\
& -\frac{v_{x}(y+\Delta y)-v_{x}(y-\Delta y)}{2 \Delta y}
\end{aligned}
$$

where $\Delta x$ and $\Delta y$ correspond to the spacing of points in the fow field calculation. We therefore calculate Div and Curl locally at each point using the four nearest points in the flow feld. Note that the difference between the Div and Curl calculations lies simply in the projection of the flow field vectors at the four nearest points; any random (noise) component of the flow field should cause identical effects in the Div and Curl calculations. We will compare the properties of Div and Curl in $\S 4$.

In calculating Div, Curl, and speed for the simulation data, we simply used the values of $v_{x}$ and $v_{y}$ provided by the simulation, rather than applying correlation tracking to the timevarying intensity field of the simulation.

There are three major sources of error in convective flows determined from the observed data. The first is noise due to nonlinear combination of distortions and scintillation that survived the destretching procedure. In unfiltered data this is by far the dominant source of noise, but it is drastically reduced in the subfundamental-filtered WL data. The second source of noise is the effect of solar p-mode oscillations. This also is present in the unfiltered GD data set but is largely eliminated in the subfundamental-filtered WL data. A third source of error arises from evolution of the granulation pattern: since correlation tracking assumes that all shifts in the crosscorrelation peak are caused by mass flows on the sun, anisotropic intensity evolution can cause errors in flows determined using correlation tracking. For the GD flow maps which were derived from unfiltered data sets, we can significantly improve the SNR by averaging a large number of adjacent frames together, at the expense of introducing some noise from evolution of features. As we show immediately below, averaging 16 successive frames significantly enhances the SNR, while preserving enough time resolution for our main purpose of testing whether the findings from the primary WL data set are affected by the filtering process.

In order to investigate the effect of the random noise, we generated a Gaussian random displacement field representing the $x$-component of the displacement derived from correlation tracking, assuming it to be pure Gaussian noise. We then derived a flow vector, $v_{x}$, by taking the difference between each pair of successive random displacement fields. We have scaled the random flow so that it has the same single-frame rms $v_{x}$ as for the GD data-namely $4.3 \mathrm{~km} \mathrm{~s}^{-1}$, i.e., we assume that this extremely high value is essentially all due to noise. We then calculated the rms of $v_{x}$ for both the GD data and the Gaussian random flow field after averaging $2^{n}$ adjacent frames together and compared their differences. Figure 1 shows the rms of $v_{x}$ for both the WL (filtered) and GD flows, and the random Gaussian flow. We note that the filtered WL flow fields have a single-frame rms $v_{x}$ of $0.8 \mathrm{~km} \mathrm{~s}^{-1}$, and the rms decreases very slowly with averaging. Averaging the GD velocity frames makes the GD rms $v_{x}$ converge onto the WL values, while averaging the Gaussian noise frames causes their rms $v_{x}$ to converge to zero. Averaging 16 irames of GD data yields an rms velocity about 2.5 times that in the 16-frame averaged Gaussian noise data; that is, a SNR of about 1.5. The following analyses of the GD data are performed on 16-frame averaged data. The WL data set, being processed by the subfundamental filter, have a very high single-frame SNR and thus are analyzed without averaging.

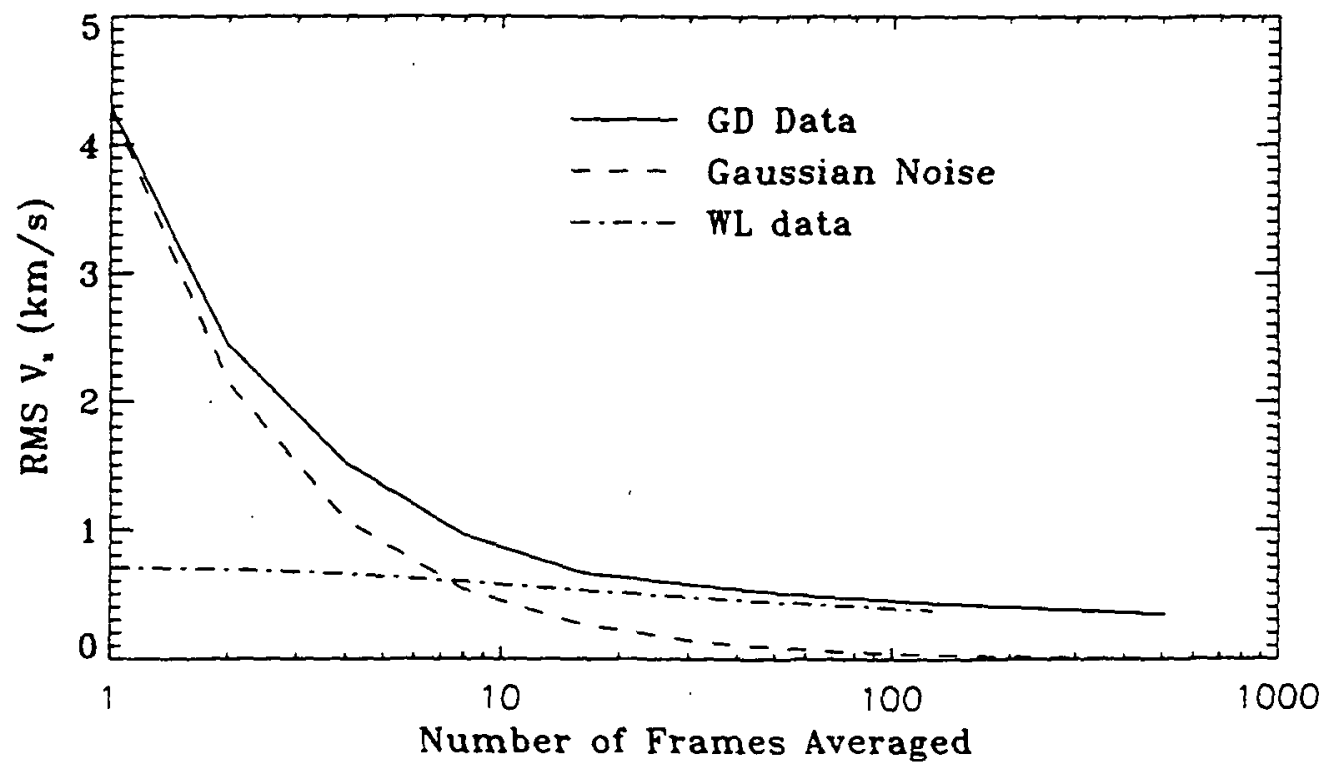

Fro. 1-Root-mean-square of $x$-component of the flow speed in the Pic du Midi GD and WL series. Averages of $2^{n}$ frames are taken before the rms calculation. The solid curve denotes the observed rms in the GD data, the short dashed line denotes the rms from a Gaussian random flow field. and the dash-dotted line shows the WL data. It is apparent from the plot that the WL series is signal dominated while averages of at least 10 frames must be taken to substantially reduce the noise level in the GD data. 


\section{GENERAL FLOW FIELD PROPERTIES}

We have found that the distribution of horizontal flow speeds as measured either from the WL flow field or the 16frame averaged GD flow field closely approximates a twodimensional Maxwellian (or Rayleigh) distribution. This is shown in Figure 2. The nonlinear best fit of a two-dimensional Maxwellian distribution to the GD distribution is plotted with the data. The most probable speed is about $0.65 \mathrm{~km} \mathrm{~s}^{-1}$. Note that the WL and GD data give essentially the same results. The slightly lower mean of the GD speed is probably caused by the decorrelation of solar signals in $320 \mathrm{~s}$ while the WL speed does not suffer from such an effect. We have calculated the same speed distribution function for the simulation data, and find that it too approximates a two-dimensional Maxwellian distribution.

A two-dimensional Maxwellian speed distribution is what would be expected if the horizontal $x$-and $y$-components of the velocity field each had Gaussian distributions. Because both the simulation and the observed flow fields show this effect, we suspect that this relationship is a real, if not obviously expected, property of turbulent convection. We note that a most probable value for the flow speed of $0.65 \mathrm{~km} \mathrm{~s}^{-1}$ could be somewhat of an overestimate, since there is still some residual noise in both flow fields.

The distribution of flow field vectors themselves as revealed by the WL data shows a gradually evolving pattern of largescale flow. Figure 3 shows the mean flow field averaged over the 57 minutes of the WL data series. The maximum mean speed is $1.25 \mathrm{~km} \mathrm{~s}^{-1}$ and the rms speed is $0.53 \mathrm{~km} \mathrm{~s}^{-1}$. It is clear that over this time span the time-averaged flow is spatially coherent. There are clearly defined mesogranular structures, as already described by Muller et al. (1992). A number of regions can be identified as having persistent outflows, such as the ones at the upper left and lower right corners in Figure 3. These regions are associated with reoccurrence of exploding granules, consistent with the findings of Simon et al. (1991). Naturally they also have large average value of Div over time. Regions of persistent outflow are also seen in the average flow fields of the GD series, indicating that they can last at least as long as 3 hours. A lifetime at least this long is not surprising, since it would take about 10 hours for mesogranules to be

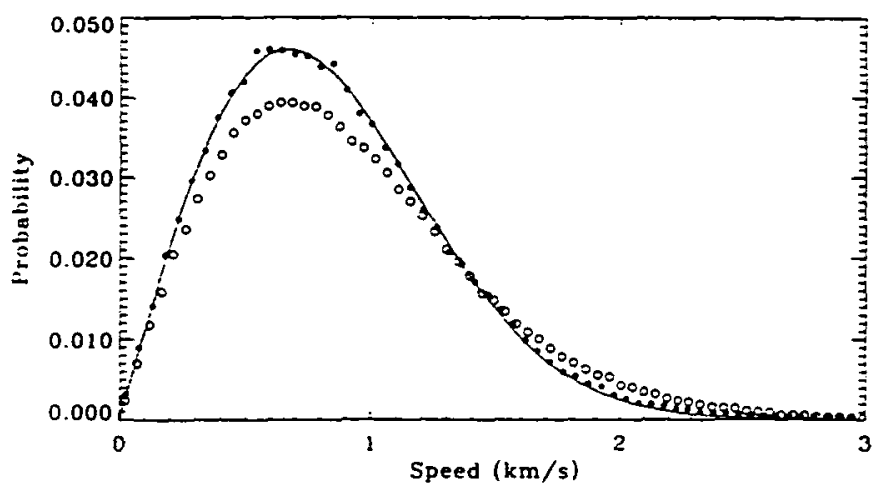

Fig. 2-Distribution of speeds in the Pic du Midi data. Dots represent the 16 frame averaged GD data while the curve represents the nonlinear best fit of a two-dimensional Maxwellian Distribution. Open circles represent the distribution of the WL data. This shows that by averaging 16 frames in the GD data. we have obtained a flow field very similar to that of the subfundamentally filtered WL data. Since the speed data is dominated by a real solar signal, we conclude that the distribution of speed in the solar photosphere closely resembles a two-dimensional Maxwellian distribution.

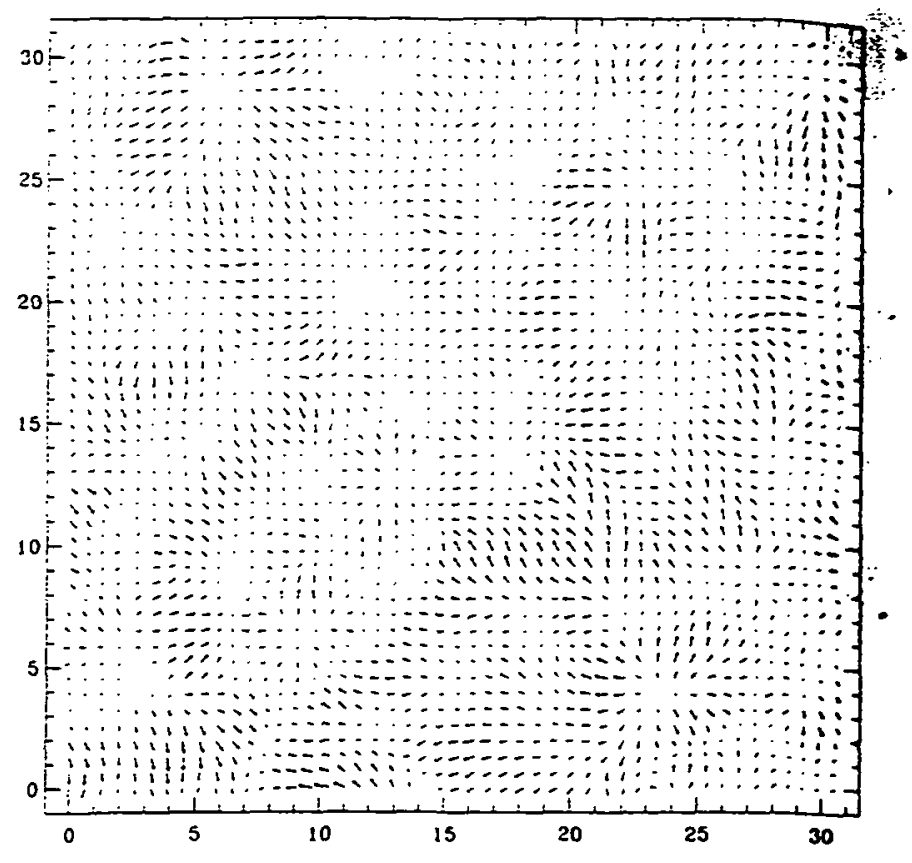

FIG. 3.-Average flow field over all adjacent pairs among the 172 frame WL data set. Each tick mark is 1 aresec. Maximum vector length corresponds to $1.25 \mathrm{~km} \mathrm{~s}^{-1}$; $\mathrm{rms}$ speed is $0.53 \mathrm{~km} \mathrm{~s}^{-1}$.

advected to supergranular boundaries and subsequently destroyed, as shown by Muller et al. (1992).

Figure 4 shows the autocorrelation function (ACF) of the velocity field $v_{x}$ for the WL data. ( $v_{x}$ is plotted here rather than speed, for later comparison with Div and Curl in the next section; all these variables have zero mean, while speed does not.) The ACF of $v_{x}$ first decreases sharply in the first two minutes and gradually stabilizes after 10 minutes. The sharp drop-off in the first 2 minutes is due to decorrelation of the noise; the 2 minute decorrelation time comes from the inherent time averaging involved in the subfundamental filtering process (Title et al. 1992). The gradual decrease between 2 and 10 minutes is mostly due to evolution of granulation over time. The stable feature after 10 minutes is due to a combination of long-lived solar features such as mesogranulation and supergranulation. This sustained ACF in time of $v_{x}$ is observed to last for at least a few hours.

\section{DIVERGENCE AND CURL}

Figure 5 shows the distribution functions of Div and Curl derived from WL flow field. Both distribution functions are Gaussian in nature, but that for Div has a clearly broader distribution than that for Curl. Essentially the same results are seen in the histograms for the GD data, where the difference between Div and Curl is even more pronounced. In the simulation data, the distribution functions for Div and Curl are also Gaussian in shape, but in contrast with the observed data sets, their widths are essentially identical.

As noted earlier, there is nothing in the numerical reduction of the data (see eqs. [1] and [2]) that is likely to introduce a systematic difference if not already pesent in the data. In principle seeing distortions can produce contributions to Div due to lensing, whereas contributions to Curl are much harder to make with wavefront errors. However, since both spatial destretching and time-averaging or temporal filtering successfully remove most of the seeing distortions, such residual effects 


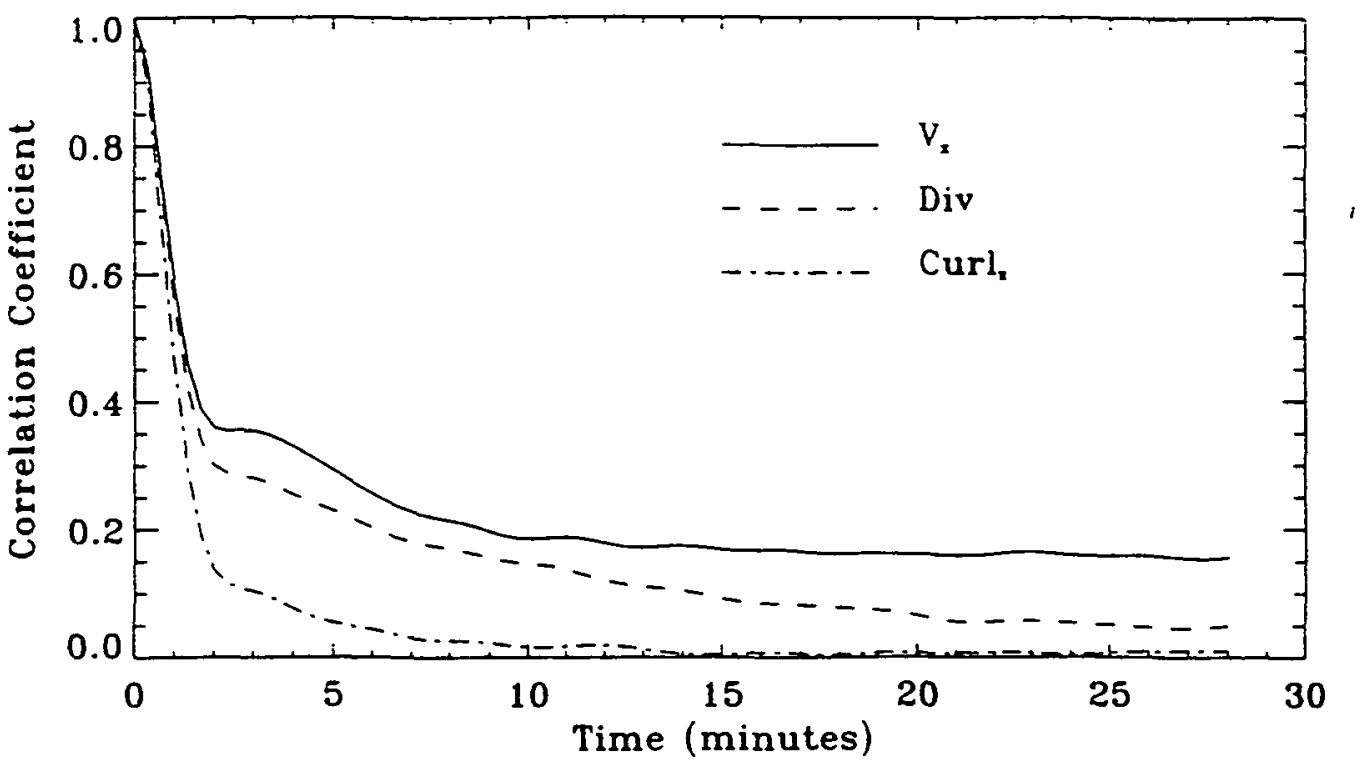

Fig. 4.-Auto-correlation function of $v_{x}$, Div, and Curl for the WL series. The sharp dropoff at the beginning is due to decorrelation of noise The coherence time of $v_{x}$ is longer than that of Div, which in tum is longer than that of Curl.

should be small. It appears more likely that we are seeing a real property of solar convection that is not captured in the convection simulations.

We have analyzed the auto-correlation function of Div and Curl along with that of $v_{x}$, as shown in Figure 4. The rapid decorrelation at the beginning of all three curves is due to noise, as already noted. In contrast to the constancy of the ACF of $v_{x}$ after 10 minutes due to steady flows at the solar surface, the dashed curve for divergence decreases steadily after 2 minutes, but remains significantly nonzero for at least 30 minutes. In contrast, the dash-dotted curve for Curl drops essentially to zero after 15 minutes. This indicates that at our resolution, Curl has a short lifetime. As we show later in this section, Curl is probably more concentrated in the intergranular lanes with a small spatial scale. We note that the ratio of the ACF of Curl to that of Div is higher for lag times of 2 to 3 minutes than it is at times exceeding 10 minutes. Possibly the contribution of intergranular lanes to the average Curl would contribute to this higher ratio for 2 to 3 minute time lags. Nevertheless, even if we scale Curl to the same level of ACF as that of Div at 2 minutes, the ACF of Curl still drops much

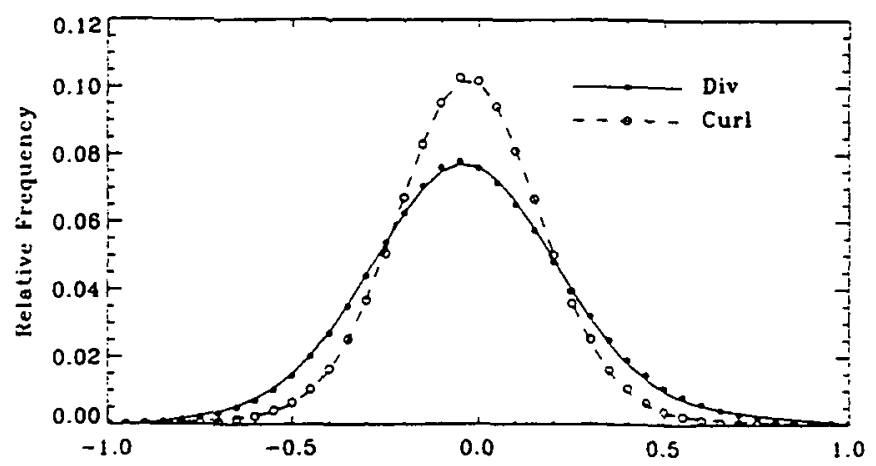

FIG. 5.-Histogram of the values of Curl (dots) and Div (circles) in the WL data set. logether with Gaussian fits to each. The distribution of Div is clearly broader than that of Curl. This is also observed in the GD data set but not in the simulation data. faster than that of Div and reaches essentially zero after 15 minutes, and thus does not change our conclusion about the difference between their lifetimes.

Figure 6 shows the spatial power spectra of Div and Curl for the WL series. The difference between Div and Curl is evident. The power of Div comes from the diverging flows in the cell centers of mesogranulation and supergranulation and converging flows in the mesogranular and supergranular boundaries. The contribution to Div associated with granules is outside the most reliable part of the data. Compared to Div, the Curl signal has relatively more power at small spatial scales corresponding to downflow regions, as established in the following paragraph. Although our data are not reliable for $k>1$ $\mathrm{Mm}^{-1}$, they nevertheless suggest that the spatial power of Curl in that range could be at least as much as that of Div. The temporal power spectra of Div and Curl have identical increasing shape with Div having more power at all values $\omega$. These results are all consistent with previously obtained results from the SOUP (Tarbell et al. 1992).

To see how Div and Curl are related to each other, we analyzed the Div and Curl data of the WL series in the following way. There are 171 frames each containing $46^{2}$ values of Div and Curl. We sorted the Div data in increasing order and divided them into 46 bins, each containing $46 \times 171=7,866$ elements. We calculated the average value of Din within each bin, $\langle\text { Div }\rangle_{\text {bia. }}$. Inside each bin, we found values of Curl corresponding to these values of Div and calculated the rms of Curl values, Curl $\mathrm{r}_{\mathrm{rms}}$. Figure 7 shows a plot of Curl $\mathrm{Cus}_{\mathrm{ma}}$ versus $\mid\langle\text { Div }\rangle_{\text {bin }} \mid$. The absolute value sign indicates the folding of results for $\langle\text { Div }\rangle_{\text {bir }}\left\langle 0\right.$ and $\left.\langle\text { Div }\rangle_{b i a}\right\rangle 0$ for plotting. Values of Curl $\left.\right|_{\mathrm{rms}}$ within bins of positive values of $\langle\mathrm{Div}\rangle_{\text {bin }}$ (called divergence) are shown with open circles and dashed lines. Solid dots and solid lines represent Curl $_{\text {rms }}$ in the negative branch $\langle\text { Div }\rangle_{\text {bin }}$ (called convergence). It is apparent in the figure that curl $_{r m s}$ has a weak tendency to be larger in regions of convergence, i.e., regions of downflow. This phenomenon is only marginally significant $( \pm 1 \sigma)$, for each pair of bins with similar $\langle\text { Div }\rangle_{\text {bio }}$; combined over all the bin pairs with $\mid\langle\text { Div }\rangle_{\text {bio }}|\rangle$ 0.10 , however, the trend is highly significant. The same asym- 


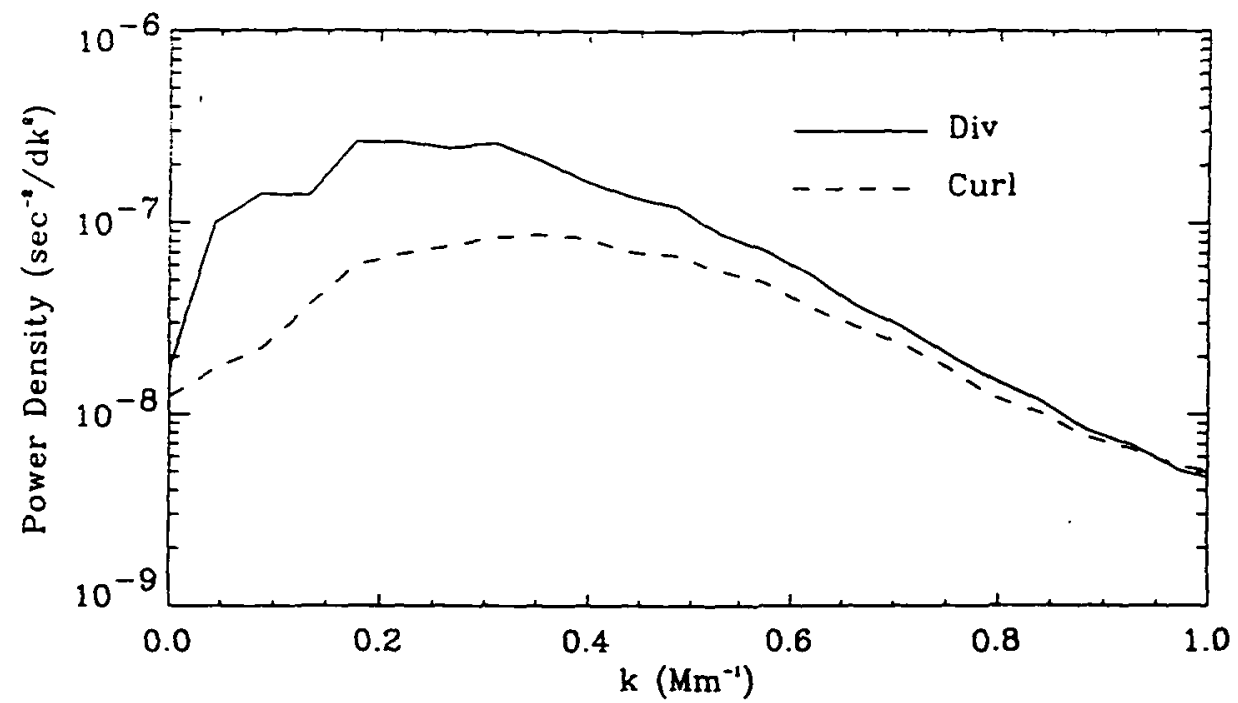

FiG. 6. - Total power density (integrated over all temporal frequencies) of Div and Curl of WL data in the spatial domain. The Div power spectrum dominates at small $k$. This is because excess power of Div is associated with clearly defined large-scale supergranular, mesogranular and granular structures, while Curl is more concentrated at downflow regions. Thus the power of Curl only matches that of Div at very high spatial frequencies. Units of power density are $s^{-2}$ per $d k^{2}=d k_{x} d k_{y}=\left(31.2 \operatorname{arcsec} \times 725 \mathrm{~km} \operatorname{arcsec}^{-1}\right)^{-2}=5.12 \times 10^{8} \mathrm{~km}^{-2}$.

metry is also present in the GD data set. The same plot for the simulation data shows the same asymmetry at least at $10 \sigma$ level in most ranges of $\mid\langle\text { Div }\rangle_{\text {bin }} \mid$ as shown in the lower half of Figure 7.

In the simulation, the Div and Curl have identical spatial and temporal power spectra, differing significantly from the observation. Thus it seems likely that something is going on in the Sun that is not present in the simulation, which gives rise to the above-mentioned differences between Div and Curl. We note again that our numerical calculations of Div and Curl from the flow field differ from each other only in the signs attached to $x$-and $y$-components of the flow vectors. Thus it is hard to see how a numerical effect could introduce a false asymmetry in the Div and Curl. While as noted, seeing could more easily introduce an instantaneous contribution to the Div field than to the Curl field, this should not affect analyses involving low temporal frequencies or large time lags in the $A C F$. Thus it appears that the different behavior of Div and Curl really represent different aspects of the physics of the flow patterns on the Sun.

We have binned the data set in bins of Curl, and made plots of Div versus $\langle\text { Curl }\rangle_{\text {bis }}$. Here we found no apparent asymmetry between Div ${ }_{\text {rms }}$ in bins with positive or negative Curl bin. This is not surprising since we do not expect a strong preferred sign of rotation at the solat surface. (Rotation-induced Coriolis forces should be negligible given the extremely small granulation and mesogranulation timescales compared to the solar rotation period.) We also plotted (Div〉 versus $\langle\text { Curl }\rangle_{\text {bin }}$ which shows a $\Lambda$ shape with roughly the same number of points above and below $\langle\mathrm{Div}\rangle=0$ line. For high values of $\mid\langle\text { Curl }\rangle_{\text {bin }} \mid$, Div is more likely to have a large negative value, i.e., strong convergence. This is consistent with the asymmetry observed in Figure 7 which also implies a stronger association between convergence and Curl than between divergence and Curl.

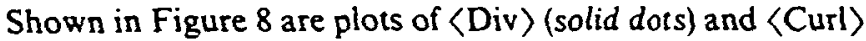
(open circles) inside bins of speed for the WL data. The solid line connects points of best polynomial fit to the Div data, while the dashed line is that to the Curl. Error bars are $\pm 1 \sigma$ of the curve fit. The two curves corresponding to the Div (solid dots) and Curl (open circles) are significantly different. At very low speed, 〈Div〉 is negative. We believe this is due to slow downflows at mesogranular and supergranular boundaries. However, 〈Curl〉 averages about zero in the low-speed region indicating no preferred sign of rotation. The general increasing trend of Div at high speed is most probably related to exploding granules whose divergence increases with speed, and whose lifetime can be as long as the time span of the data set. (Curl) remains near zero, once again indicating no preferred sign of rotation. The highest points in both curves are clipped off to keep the noise level down. The 16 frame averaged GD data produces an almost identical graph.

\section{ROTATIONAL MOTION OF BRIGHT PONTS}

$A$ large number of sub-arcsecond bright points can be found in high-resolution continuum images. They tend to cluster around the supergranular boundaries (Muller et al. 1992). They are typically 0.5 aresec in size or smaller with mean intensity higher than that of granular centers. Most of these bright points live on the order of a few minutes. However, some can live much longer. We have found a number of bright points that persisted over 30 minutes. They are undoubtedly closely related to the magnetic field. However, the true nature of NBPs awaits future high resolution continuum images with correlated magnetic field data.

We searched for long-lived bright points using space-time slices. They are obtained by slicing the three-dimensional data array in the spatial-temporal plane rather than the $x-y$ plane. The upper part of Figure 9 (Plate 15) shows one of these slices. Granules appear to be bright, elongated broad regions, while bright points appear as thin bright feature surrounded by thin dark lanes. The time slices show occasional wavy bright features within what appear to be intergranular lanes-suggestive of a bright point moving back and forth in $x$, either due to a horizontal oscillation or perhaps due to circular motion such as a vortex.

Through careful manual screening of the frames, we found that rotational motions of small bright points do occasionally 

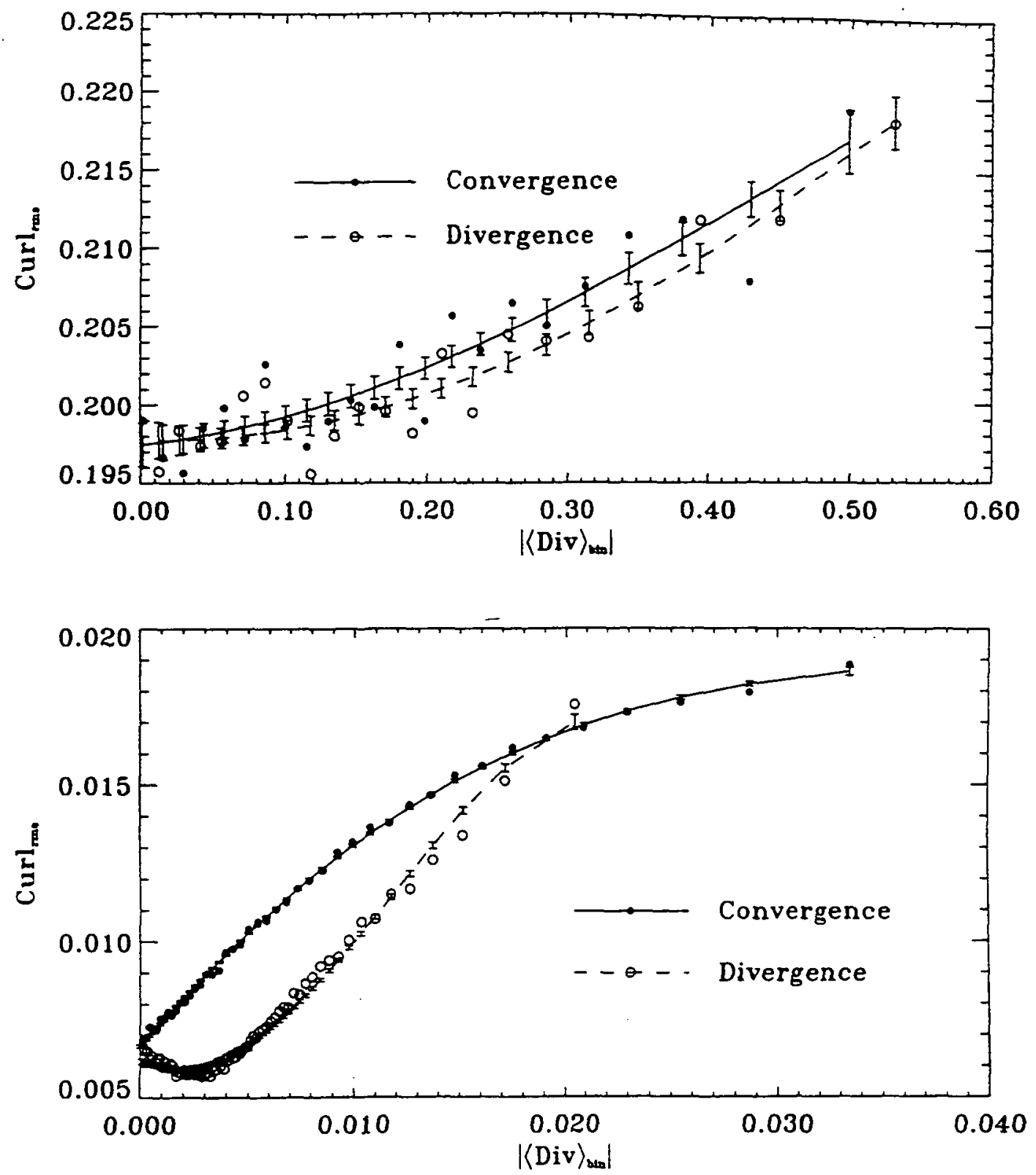

Fig. 7.-Plots of Curl rat $_{\text {v. }} \mid\langle\text { Div }\rangle_{\text {bin }} \mid$ for the WL data (top) and the numerical simulation data (bottom). In each bin of Div we calculate the average value of Div and the ms of the values of Curl associated with these points. We have folded the axis around zero for display. Solid dots represent Curl solid lines indicating the best cubic polynomial fit, and error bars indicating $\pm 1 \sigma$ error to the fit. Open circles and the dashed lines are for Curl The largest value in Curl ros $_{\text {for }}$ both curves has been clipped because they are dominated by noise.

occur. An outstanding example is shown in Figure 9, where two small bright points, separated by about 0"65 or $450 \mathrm{~km}$, are seen to rotate about each other at a speed of about 3 $\mathrm{km} \mathrm{s}^{-1}$, corresponding to an angular velocity about 1 radian in $160 \mathrm{~s}$, or a period of about $1000 \mathrm{~s}$. The vorticity in this case is about 9 times that of the large vortex observed by Brandt et al. (1988), at $1.3 \times 10^{-2} \mathrm{~s}^{-1}$. However, these rapid rotations are at the limit of our resolution. Numerical simulation indicate that vortex motion is common in downflow regions surrounded by granules though without bright points as tracers, they would not be easily seen. The trend toward large vorticities at small spatial scales is also consistent with the results derived from the medium and low spatial resolution analysis by Simon et al. (1994).
Such clearly defined events proved hard to find. In fact visual search of the WL images resulted in only about a dozen clear bright points whose identity clearly persisted over more than 10 minutes. Most of the small bright points were found to bifurcate or simply disappear after a few minutes, with no clear sign of rotation. We have only one other example where rotation between two bright points can be seen. One problem is the definition of a coordinate system that persists from frame to frame; slight displacements of the frames (even given the prealignment of the frames) can mask motions at the sub-arcsec level. Thus isolated NBP are not a good tracer of local motions because of lack of a good rigid coordinate system. The bright point pair shown in Figure 9 avoids the difficulty, since we can measure relative motions between the two bright points. 


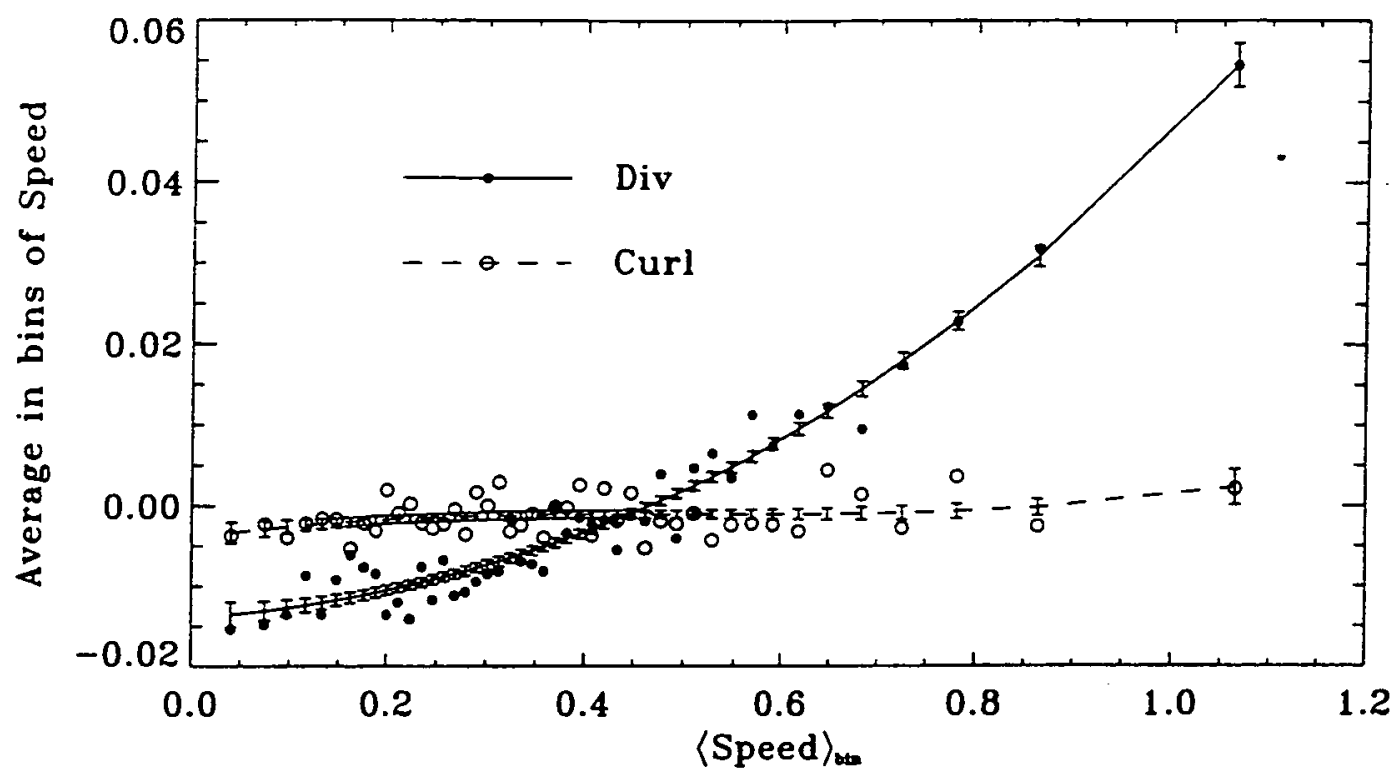

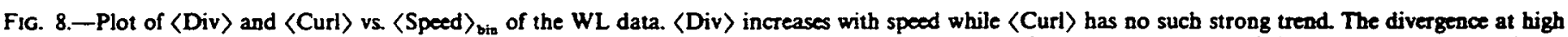
speed is thought to be caused by regions of exploding granules where both speed and Div are large. Some of these regions have a lifetime at least as long as 1 hour. The 〈Div〉 goes below zero at low speed while 〈Curl〉 does not. This difference is probably related to downflow regions at granular, mesogranular, and supergranular boundaries, where convergence is preferred while Curl has no preferred sign.

Another difficulty in studying NBPs arises from the complexity of the dynamics in the field being observed. Motions of granules, exploding granules, surface waves, and other events make it difficult to track these small bright points and their identities are often confusing over time. Space-time slices are very helpful in tracing NBPs. While the bright points can be unclear in image space, the continuity in space-time slices helps preserving their identity.

\section{DISCUSSION AND CONCLUSION}

The above analyses have shown that Div and Curl of horizontal flow fields in the solar photosphere have distinct characteristics. In summary, we note the following observations.

1. Vorticities seem to have a closer association with inflows than outflows. The asymmetry in our $\mathrm{Curl}_{\mathrm{rms}}$ versus $\left|\langle\mathrm{Div}\rangle_{\text {bin }}\right|$ plot indicates this. This finding is confirmed by the filtered, averaged unfiltered, and simulation data. This extends but is consistent with results others have obtained. Brandt et al. (1988) found a large vortex associated with an inflow region which lasted a few hours. Using a superb set of data from La Palma, Simon et al. (1994) found that at large spatial scales, Curl is associated with downflow regions, and at large temporal scales, vortices can last as long as several hours. Zirker (1993) also found prevalent vorticities in the intergranular lanes, from study of solar convection simulations.

2. Spatial power spectrum of Curl shows higher amplitudes at smaller spatial scales compared with that of $\mathrm{Div}$, and temporal autocorrelation shows that the coherence time of the Curl field is significantly shorter than that of Div. Both numerical simulation and observation of bright points convince us of the fact that the strong vortices exist at granular boundaries, where the spatial and temporal scales are small. The ratio of spatial and temporal power of Curl over Div grows toward smaller spatial and temporal scales, supporting this conclusion. Simon et al. (1994) also observed more power of Curl on smaller scales.

3. High speed is associated with high outflow. We interpret this as the relationship between exploding granules and high divergence. Simon et al. (1991) have pointed out that areas of persistent exploding granules are mesogranular structures seen as diverging regions in time averaged flowmaps. At low speed, we observe convergence which is thought to be associated with inflow at meso- and supergranular scales. Curl has no preferred sign in either high or low speeds, thus averaging to zero.

4. Large local vorticities have been observed. The specific case described here has vorticity 9 times that of Brandt et al. (1988) and 50 times that of Simon et al. (1994). The spatial and temporal scales increase from our observation to that of Brandt, and then to that of Simon. This is consistent with the predictions above.

5. Although in the numerical simulation, the shape of the spatial and temporal power spectra of Div and Curl are essentially the same, they are significantly different in the observed data.

Although not discussed in the text, we have analyzed a set of La Palma data of coarser resolution in exactly the same way as described previously and found the same conclusions as above.

This project was supported by NASA grant NAGW-2545 and the Ford Program for Undergraduate Research at Harvard University; and by NASA contract NASW -4612 and the Independent Research Program at Lockheed. We are very grateful to R. Muller who acquired the outstanding Pic du Midi data set, and to $D$. H. Porter for providing the simulation data. We thank Neal Hurlburt for useful comments about the simulation data. Y. W. thanks Richard Shine, Zoe Frank, and others at LPARL for their hospitality and assistance during his visit. 


\section{REFERENCES}

Brandt. P., Scharmer, G., Ferguson, S., Shine, R., Tarbell, T., \& Title. A. 1988 Nature. 335.238

Muller. R. 1990, in Solar Photosphere: Structure, Convection, and Magnetic Fields, ed. J. O. Stenflo (Dordrecht: Kluwer, 85

Muller. R., Auffret, H., Roudier, T., Vigneau, J., Simon, G., Frank, Z., Shine, R. \& Title, A. 1992, Nature, 356, 322

Muller, R., \& Roudier, T. 1992 Sol. Phys., 141, 27

vovember, L. J. 1986, Appl. Opt_ 25, 392

November, L. J., \& Simon, G. W. 1988, ApJ, 333, 427

Porter. D. H. Woodward, P. R. \& Mei, Q. 1991, University of Minnesota Supercomputer Institute Research Report Number UMSI 91/150 (Minneapolis: Univ. Minnesota)

Simon, G. W. Brandt, P. N., November, L. J., Scharmer, G. B. \& Shine, R. A 1994, in Solar Surface Magnetism, od. R. Rutten \& C. Schrijver (Dordrecht: Kluwer, 261

simon, G. W. Title, A. M. Topka, K. P. Tarbell, T. D., Shine, R. A., Ferguson,

S. H. Zirin, H. \& the SOUP Team. 1988, ApJ, 327, 964

Simon, G. W. Title, A. M. \& Weiss, N. O. 1991, ApJ, 375, 775

Sturrock. P. A. \& Uchida. Y. 1981, ApJ, 246. 331

Tarbell, T. D.. Slater. G. L.. Frank, Z. A. Shine, R. A. \& Topka, K. P. 1992 in Mechanisms of Chromospheric and Coronal Heating, $\propto d$. P. Ulmschneider. R. Rosner, \& E. Priest (Berlin: Springer), 39

Title, A., Tarbell. T., Simon, G. \& the SOUP Team. 1986. Adv. Space Res, 6, 253

Title A. M. Tarbell T. D. Topka, K. P., Ferguson, S. H, Shine, R. A \& the SOUP Team. 1989, ApJ, 336, 475

Title, A. M. Topka, K. P., Tarbell, T. D., Schmidt, W, Balke, C, \& Scharmer, G. 1992 ApJ, 393, 782

Topka, K. P. Tarbeli, T. D, \& Title, A. M. 1986, ApJ, 306, 304

Zirker, J. B. 1993. Sol. Phys., 147, 47

Zirker, J. B., \& Cleveland. F. M. 1993, Sol. Phys., 144, 341 